\title{
Clinical Study \\ The Effect of Kinesiology Taping on the Hemiplegic Shoulder Pain: A Randomized Controlled Trial
}

\author{
Lin Yang, Jingyi Yang $\mathbb{D}^{\mathbb{D}}$, and Chengqi He \\ The Rehabilitation Department of the West China Hospital, Sichuan University, Chengdu, China \\ Correspondence should be addressed to Chengqi He; hxkfhcq@126.com
}

Received 10 November 2017; Accepted 11 November 2018; Published 10 December 2018

Academic Editor: Emiliano Schena

Copyright (c) 2018 Lin Yang et al. This is an open access article distributed under the Creative Commons Attribution License, which permits unrestricted use, distribution, and reproduction in any medium, provided the original work is properly cited.

Objective. The purpose of the study was to explore the effect of kinesiology taping on hemiplegic shoulder pain (HSP) in terms of pain intensity, magnitude of subluxation, muscle activity, and active range of motion (AROM). Design. Double-blind, placebo-controlled clinical trial. Setting. the Rehabilitation Center of the West China Hospital. Participants. Nineteen individuals suffering from HSP were recruited in this study. Intervention. Patients were randomly assigned into the taping group or control group. The taping group received therapeutic kinesiology taping and conventional treatment, while the control group received placebo taping (applied without tension) and conventional treatment. Main Outcome Measures. The shoulder pain intensity (numerical pain rating scale), magnitude of subluxation, muscle activity (measured by surface electromyography (sEMG)), and shoulder active range of movement (AROM) were assessed at the baseline, on the first day (immediately after taping) and 4 weeks after treatment (without taping). Results. All patients completed the trials. There were no significant differences between groups at the baseline. The taping group showed immediate improvement on the first day after taping in terms of pain intensity, magnitude of subluxation, and muscle activity $(p<0.05)$, whereas no significant changes seen in the control group $(p>0.05)$. After 4 weeks of treatment, the taping group showed significant changes in pain intensity, magnitude of subluxation, muscle activity, and AROM $(p<0.05)$. And significant differences in pain intensity and muscle activity could be seen between the two groups $(p<0.05)$. Conclusion. The results indicate that the kinesiology taping is effective in reducing the shoulder pain and subluxation and increasing muscle activity and AROM for patients with HSP after stroke.

\section{Introduction}

Shoulder pain is a common complication in hemiplegic patients after stroke. The incidence of hemiplegic shoulder pain (HSP) is reported to be approximately $17-72 \%[1-6]$. HSP can inhibit the muscle contraction and limit efforts of patients to conduct exercise and delay the recovery in the motor function and influence capacity of performing daily activities and social participation [7-9]. This may further increase the length of a patient's stay in the hospital [10].

The mechanisms for HSP are not fully understood. Muscle weakness after stroke is believed to be a primary reason for HSP. As the shoulder muscles cannot contract effectively against gravity and external force during movement, the humeral head cannot be held in a proper position. Soft tissues around the shoulder may be gradually stretched and torn, resulting in HSP [11]. This understanding constitutes a reasonable mechanism to explain why soft tissue injuries are usually observed in HSP [12-15]. Other factors such as disturbed sensory and cognitive function are also proved to be involved in HSP [16].

Regardless of the limited understanding of the etiology of HSP, various therapeutic methods have been trialled to examine their effects on HSP. Different types of slings and strapping methods which can provide effective support to the shoulder are usually involved in the therapeutic strategy for HSP $[6,17]$. Electrical therapy and appropriate exercise program are also proved to be necessary for the treatment of HSP [18-21]. However, evidence for the effectiveness of these conventional methods for HSP is limited. And some of these treatment methods have significant problems and limitations to their use, such as sling and strapping which 
may limit the movement of the shoulder and interfere with the recovery of the function $[18,21,22]$.

Kinesiology taping, sometimes also called elastic taping, is a relatively new technique used in rehabilitation programs. This method is different from strapping or rigid taping technique. The elastic tape is a hypoallergenic skin tape with elastic components. This kind of elastic tape is designed to allow for a longitudinal stretch of $55-60 \%$ of its original extent. By using the kinesiology tape, different stretches and directions can yield different effects for reducing pain, correcting alignment, facilitating neuromuscular activity, and alleviating inflammation and edema [23-25]. Based on these notions about the features of kinesiology taping, it can be hypothesized that kinesiology taping can be used for patients with HSP to reduce the pain, correct the subluxation, activate the muscles of shoulder, and further restore the motor function. However, few studies have been done to provide evidence for the hypothesis. Therefore, the aim of this study is to investigate the effects of kinesiology taping on HSP.

\section{Methods}

2.1. Research Design. A double-blind, placebo-controlled clinical trial was carried out to investigate the effects of kinesiology taping on HSP. The blind method was used for the patients and therapists who provided conventional treatments and assessment. The primary outcome measurement was set as the pain intensity of the shoulder, and the secondary outcome measurements included the magnitude of subluxation, active range of motion (AROM), and muscle activity of the shoulder.

The study protocols were approved by the Ethics Committee of the West China Hospital of Sichuan University in China and were funded by the Sichuan Provincial Department of Public Health. And all participants provided written informed consent.

2.2. Participants. The trial was carried out in the Rehabilitation Center of the West China Hospital from April 2013 to September 2014. Nineteen participants with a diagnosed first-ever stroke were recruited to the study. All participants met the following inclusion criteria: $(1)>30$ years of age; (2) period after stroke: $>1$ month and $<6$ months; (3) diagnosed as HSP with a period of more than 1 month, accompanied with shoulder subluxation; (4) adequate communication abilities; (5) the shoulder muscles can contract and move the shoulder more than 10 degrees but less than 90 degrees in flexion and/or abduction in sitting position, accompanying shoulder pain produced or increased; (6) normal light touch and pin-prick sensation on the affected shoulder; (7) the pain is caused by local problems: (1) manual palpation and movement can affect the pain and (2) reposition of the joint can relieve the pain.

The exclusion criteria include (1) history of serious conditions or diseases such as cancer; (2) skin problems, wounds, or infections on the affected shoulder; (3) skin allergy to the tape; (4) history of shoulder fracture on the affected side or history of shoulder sprain or subluxation before the stroke; (5) severe disease which may affect the study, such as uncontrolled hypertension or heart disease; (6) history of intra-articular steroid injection in the past 4 weeks.

2.3. Procedure. According to the recruited order and the corresponding random number sealed in an envelope, patients were assigned into the taping group or the control group. Before treatment, the demographic data and clinical characteristics of the participants were recorded. The outcome measures were assessed at the following time points: at the baseline (one day before the treatment), on the first day (immediately after taping on the first day, with taping applied), and after 4 weeks (on the following day after the 4 weeks treatment completion, without taping).

All the participants received treatment once a day, 5 days per week for 4 consecutive weeks. The treatment program included kinesiology taping, electrical therapy, and exercise treatment. Electrical therapy was applied first, followed by taping and finally exercise. The therapeutic taping was used for the taping group, while placebo taping (without tension) was applied for the control group.

Exercise therapy for the affected shoulder included passive and active assistant and active exercises. These exercises focused on restoring and improving the motor function of the shoulder. The range of motion (ROM) exercise of the affected shoulder was demanded to be less than 90 degrees to avoid potential injury to the joint. The exercise time for the affected shoulder was $20 \mathrm{~min}$. The patients also received 40 mins exercise for the trunk and low limb.

Electric therapy (Bengao-computerized intermediatefrequency therapy apparatus, Beijing Bengao High technology Co. Ltd.) was applied in the study. The electrodes were placed over the posterior and anterior aspects of the shoulder. The pain-reduce program was chosen for all patients. The treatment time was set to $20 \mathrm{~min}$, and the output current was set to $20-40 \mathrm{~mA}$.

The therapeutic taping aimed to activate the neuromuscular function and produce mechanical support to the shoulder. Tapes of $5 \mathrm{~cm}$ width were used for patients. The taping method is shown in Figure 1. The facilitation technique was used for the deltoid, supraspinatus, and teres minor. First, the supraspinatus was taped. The shoulder was positioned in an abduction potion at about 30 degrees with a slight flexion and internal rotation, and the humeral head was repositioned to the normal place. The first $4 \mathrm{~cm}$ of the tape was applied to the original site of supraspinatus (superior medial border of the scapula) with no tension. Then, the remaining strip was applied over the muscle to the insertion site (greater tubercle of humerus) with about $25-50 \%$ of the full available tension. After this, the patient's shoulder was placed in abduction at 30 degrees. Taping of the middle part of deltoid muscle begun by attaching the first $4 \mathrm{~cm}$ of the strip over the acromion process with no stretch. Then, the rest of the strip was stretched downward to the deltoid tuberosity with $20-30 \%$ of tension. For taping the teres minor, the shoulder was flexed with a little internal 


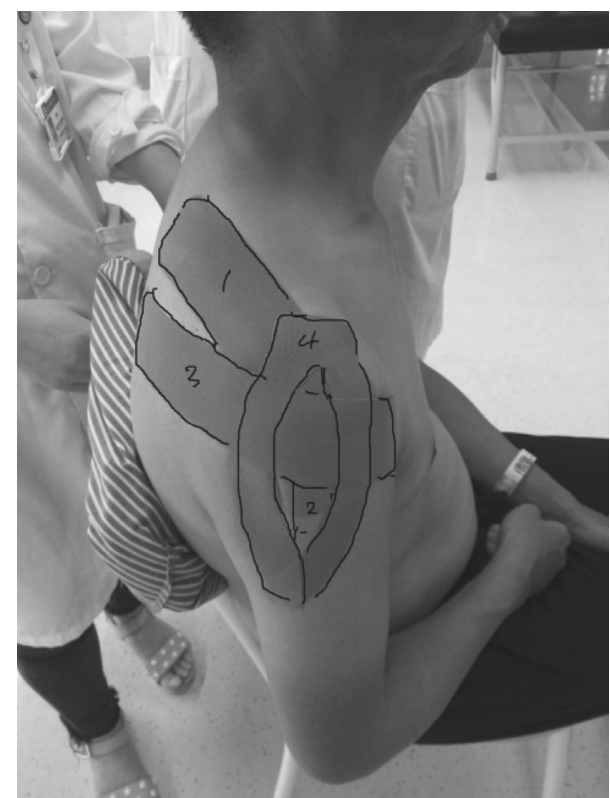

FIGURE 1: Kinesiology taping for patients. (1) supraspinatus; (2) middle part of deltoid; (3) teres minor; (4) anterior and posterior part of deltoid.

flexion. The base of the tape was placed on the inferior angle of scapular. The rest of the strip was stretched with 15-25\% of tension and placed along the axillary border of the scapula to the greater tuberosity of the humerus. The last one tape was used to reduce the subluxation of the shoulder and was cut into Y shape before taping. After reposition of the shoulder, the base of the tape was applied to the acromion process, and then, the two strips were stretched with a tension of $50-70 \%$ and placed along the anterior and posterior borders of deltoid separately to the deltoid tuberosity. For the placebo group, the tapes were applied on the same place but no tension was applied. The tapes were removed at about $8 \mathrm{pm}$. The time for taping was ensured to be 10 to 12 hours per day. After removing the tape, the skin was cleaned with warm water to keep it healthy.

\subsection{Outcome Measures}

2.4.1. Pain Intensity. Shoulder pain was the primary outcome measure and was assessed by a numerical pain rating scale (NPRS) which has a high correlation coefficients with the visual analog scale and is simple to use [26]. The pain intensity is graded from 0 to 10 where 0 means no pain and 10 means the most intense pain. The maximum pain during shoulder flexing and abducting in sitting position was recorded.

2.4.2. Magnitude of Shoulder Subluxation. The patient was kept unsupported in an upright posture without a backrest or armrests, with the arms in a neutral position hanging by the side of the body. The inferior border of the acromion and the highest of greater tubercle of the shoulder was palpated and marked. The vertical distance between the two points was measured with a tape measured and recorded. The difference of the distance between the affected and normal sides was defined as the magnitude of subluxation.

2.4.3. AROM of the Shoulder. In a sitting position, the patient was instructed to first flex and then to abduct the affected shoulder as much as possible. The AROM of the affected shoulder in flexion and abduction was measured with the goniometer and recorded.

2.4.4. Muscle Activity. Electrical sensors have emerged as an efficient way to characterize biomedical signals. The muscle activity was assessed with sEMG. The Muscle Tester ME6000 (Mega Electronics Ltd., Kuopio, Finland) was used for the purpose of the study. The muscle electrical activation of the middle part of deltoid and supraspinatus muscles during shoulder abducting in sitting position was recorded. Prior to the test, the skin was cleansed with 75\% alcohol. Disposable $8 \mathrm{~mm}$ AgCI surface electrodes (Blue sensor, Ambu, Denmark) were attached over the middle part of deltoid and supraspinatus muscles on the affected side. Sampling of the muscle activity was done at a frequency of $1000 \mathrm{~Hz}$. The data were downloaded to an EMG analyzer, Megawin 2.5 (Mega Electronics Ltd., Kuopio, Finland), where the data were analyzed. The average amplitude, which was presented with AEMG $(\mu \mathrm{V})$, was calculated for further analysis.

2.5. Data Analysis. Statistical analysis was carried out by using the SPSS version 20.0. The results were presented as mean (SD). The chi-square analysis was used to determine the group difference in sex and side of hemiplegia. Paired sample $t$-test and independent sample $t$-test were applied to examine the difference within group and between the two groups, respectively. The significance level was set at $p<0.05$.

\section{Results}

Totally, 19 patients were recruited in the study. The demographic characteristics and measurements at the baseline are shown Table 1 . There were no significant differences between the two groups at the baseline. All the participants completed the whole trial, and there were no side effect observed.

Based on the data listed in Tables 2-5, the taping group showed an immediate and significant improvement in pain intensity, magnitude of subluxation, and AEMG on the first day immediately after taping, compared with the baseline $(p<0.05)$, whereas there was no significant changes in the control groups in these outcomes. Both groups showed no significant changes in the AROM in flexion and abduction on the first day $(p>0.05)$. After 4 weeks of treatment, significant improvement was seen in the taping group in terms of pain intensity, magnitude of subluxation, AEMG, and AROM (both flexion and abduction). Based on the baseline, the amount of improvement in the pain intensity, 
TABLE 1: Baseline characters of patients in the two groups.

\begin{tabular}{lccc}
\hline & Taping group & Control group & $p$ value \\
\hline Number & 10 & 9 & \\
Age (years) & $59(3.2)$ & $60(2.3)$ & 0.939 \\
Male : female & $7: 3$ & $6: 3$ & 0.875 \\
Left : right (hemiplegia) & $4: 6$ & $5: 4$ & 0.498 \\
Time poststroke (weeks) & $18.3(0.82)$ & $19.2(2.49)$ & 0.502 \\
Pain & $4.3(1.2)$ & $5.0(0.7)$ & 0.111 \\
Subluxation (cm) & $1.22(0.39)$ & $1.18(0.33)$ & 0.807 \\
ROM (F) (degrees) & $27.8(6.1)$ & $31.7(7.6)$ & 0.238 \\
ROM (Abd.) (degrees) & $24.8(5.5)$ & $25.6(6.8)$ & 0.763 \\
AEMG (S) $(\mu \mathrm{V})$ & $152.6(27.9)$ & $163.3(33.1)$ & 0.925 \\
AEMG (D) $(\mu \mathrm{V})$ & $126.8(11.5)$ & $134.6(10.4)$ & 0.482 \\
\hline
\end{tabular}

Note. $\mathrm{F}=$ flexion; $\mathrm{Abd} .=$ abduction; $\mathrm{S}=$ supraspinatus muscle; $\mathrm{D}=$ deltoid muscle.

TABle 2: Pain intensity assessed at different time points.

\begin{tabular}{lcccc}
\hline & $n$ & Baseline & First day & 4 weeks later \\
\hline Taping group & 10 & $4.3(1.2)$ & $0.6(0.6)^{* \wedge}$ & $1.4(0.7)^{* \wedge}$ \\
Placebo group & 9 & $5.0(0.7)$ & $4.8(0.8)^{*}$ & $3.4(0.8)^{* \wedge}$ \\
$p$ value & & 0.111 & 0.000 & 0.000 \\
\hline
\end{tabular}

${ }^{*}$ Independent samples $t$-test, compared between groups, $p<0.05$; ${ }^{\wedge}$ paired sample $t$-test, compared between before and after treatment, $p<0.05$.

TABle 3: Magnitude of subluxation $(\mathrm{cm})$ of the shoulder.

\begin{tabular}{lccc}
\hline & Baseline & First day & 4 weeks later \\
\hline Taping group & $1.22(0.39)$ & $0.71(0.20)^{* \wedge}$ & $0.91(0.31)^{\wedge}$ \\
Control group & $1.18(0.33)$ & $1.18(0.31)^{*}$ & $1.18(0.26)$ \\
$p$ & 0.807 & 0.01 & 0.062 \\
\hline
\end{tabular}

${ }^{*}$ Independent samples $t$-test, compared between the taping group and the control group, $p<0.05$; $\wedge$ paired sample $t$-test, compared between before and after treatment, $p<0.05$.

TABle 4: The AEMG $(\mu \mathrm{V})$ tested at different time points.

\begin{tabular}{lcccc}
\hline \multirow{2}{*}{ Group } & \multirow{2}{*}{ Muscles } & \multicolumn{3}{c}{ AEMG $(\mu \mathrm{V})$} \\
& & Baseline & First day & 4 weeks later \\
\hline \multirow{3}{*}{$\begin{array}{l}\text { Taping } \\
\text { group }\end{array}$} & \multirow{2}{*}{ Deltoid } & 126.8 & 140.0 & 221.6 \\
& & $(11.5)$ & $(13.9)^{\wedge}$ & $(34.7)^{* \wedge}$ \\
& \multirow{2}{*}{ Supraspinatus } & 152.6 & 177.0 & $273.3(37.7)^{* \wedge}$ \\
& & $(27.9)$ & $(33.1)^{\wedge}$ & \\
Control & \multirow{2}{*}{ Deltoid } & 134.6 & 135.7 & 167.78 \\
group & & $(10.4)$ & $(12.9)$ & $(10.2)^{* \wedge}$ \\
& \multirow{2}{*}{ Supraspinatus } & 163.3 & 161.3 & 198.1 \\
& & $(33.1)$ & $(35.6)$ & $(33.7)^{* \wedge}$ \\
\hline
\end{tabular}

${ }^{*}$ Independent samples $t$-test, compared between the taping group and the control group, $p<0.05 ;{ }^{\wedge}$ paired sample $t$-test, compared between before and after treatment, $p<0.05$.

magnitude of subluxation, AEMG, and AROM were significantly greater than the control group $(p<0.05)$.

The statistical power was calculated by using the software $\mathrm{G}^{*}$ Power. The calculation was based on the primary measure, NPRS. The power is 0.99 which indicates that power level is acceptable, and the sample size is enough for this study.
TABLE 5: The AROM of the shoulder (degree) assessed at different time points.

\begin{tabular}{lcccc}
\hline Group & & Baseline & First day & 4 weeks later \\
\hline \multirow{2}{*}{ Taping group } & Flexion & $27.8(6.1)$ & $28.8(6.2)$ & $40.1(6.8)^{\wedge}$ \\
& Abduction & $24.8(5.5)$ & $26.7(5.4)^{\wedge}$ & $33.4(5.1)^{\wedge}$ \\
\hline \multirow{2}{*}{ Control group } & Flexion & $31.7(7.7)$ & $31.7(7.7)$ & $37.9(9.6)^{\wedge}$ \\
& Abduction & $25.6(6.8)$ & $26.0(6.9)$ & $29.8(9.2)^{\wedge}$ \\
\hline
\end{tabular}

${ }^{*}$ Independent samples $t$-test, compared between the taping group and the control group, $p<0.05$; ${ }^{\wedge}$ paired sample $t$-test, compared between before and after treatment, $p<0.05$.

\section{Discussion}

To our knowledge, this is the first study aimed at exploring the effects of kinesiology taping on HSP in terms of pain, shoulder subluxation, AROM, and muscle activity of the shoulder. The results indicated that kinesiology taping is an effective method for HSP management.

It has been noticed that some other types of taping methods were trialled to examine their effect on HSP. Despite the difference in tapes and taping methods, most of these studies focused on the effect of taping on preventing the development of HSP [27, 28]. As far as we know, few studies examined the effectiveness of kinesiology taping in treating HSP.

Actually, kinesiology taping has been widely used to treat musculoskeletal problems. Its effects on nonspecific pain, such as neck pain, impinged shoulder pain, and knee pain, have also been examined. In the present study, a significant reduction of pain (NPRS, from 4.3 points to 0.3 points, $p<0.05)$ was observed immediately after taping was applied on the first day. The immediate effectiveness of kinesiology taping in modulating pain was reported in previous studies focusing on treating impinged shoulder pain, neck pain, and low back pain $[25,29-31]$. However, the mechanism for the immediate effect is still unknown. One of the proposed mechanisms suggests increasing the afferent feedback to the spine. Under the gate control theory, then increase in afferent stimulus can reduce the conducting of nociception into the central nervous system [32]. In addition, the effect of alignment correction may be another potential mechanism. The alignment correction effect on skin and shoulder posture has been proven $[33,34]$. The present study also showed notable effectiveness in reducing the shoulder subluxation. It is reasonable that the reduced subluxation can decrease the stimulation to nociception sensors, resulting in pain modulation. Different from the immediate effect on pain modulation, the reduced pain observed after 4 weeks could be ascribed to healing because when the assessment at week 4 posttreatment was done without taping.

Besides the reduction in pain intensity, kinesiology taping also yielded notable decrease of subluxation. Even though the reliability and validity of the method for assessing the subluxation have not been examined, considering the blind method was applied to the assessor and patients, the results have credibility for discussion. The alignment correction effect of taping has been widely examined and accepted [34, 35]. Reducing the subluxation 
can provide an opportunity to injured tissue to heal. The effectiveness observed after 4 weeks can be ascribed to the enhanced healing process. Based on the finding, it can be concluded that kinesiology taping can enhance the healing of injured tissue around the shoulder. Similarly, a sling can also provide mechanical support to the subluxed shoulder [36]. Previous studies indicated that a sling is able to bring benefit to patients with HSP in shoulder subluxation [36] and walking efficiency [37]. However, a sling may limit the movement of the shoulder. Most importantly, the sling usually places the arm anteriorly. This may aggravate the flexion pattern of upper extremity and increase the scapular lateral rotation pattern which has been proved to be linked with HSP [38]. This may be one of the reasons why sling treatment leaded to a conflicting result in previous studies [18]. Strapping may be another alternative to provide support to the shoulder. However, the rigid tape cannot work effectively during movement. A study demonstrated that 15-20 min of exercise can cause the rigid tape losing its function to restrict joint [39]. In contrast with strapping, kinesiology taping can provide effective support to the shoulder. Its elastic quality conforms to the body and allows for movement.

In addition, the muscle activation effect of kinesiology taping has been discussed and studied. Macgregor et al. designed a study to investigate the effect of taping on muscle activity in people with patellofemoral pain. Results demonstrated that stretching to the skin via taping can increase muscle activity [40]. Some other recent studies also provided evidence suggesting that taping can affect the muscle activity $[41,42]$. However, the muscle activation effect of taping was not supported by the study conducted by Ryan and Rowe in which the symptomatic participants did not show significant changes in surface electromyography indices after taping [43]. In the present study, facilitation technique has been applied to the middle part of deltoid, supraspinatus, and teres minor. Data showed significant improvement AEMG in the taping group after taping, implying that kinesiology taping can activate the muscles. However, the increased AEMG may also be contributed to the reduced inhibition of pain to muscles and alternated kinesiology induced by increased subluxation. The muscle activation effect might therefore be a result of multiple mechanisms.

Despite the immediate improvement in pain, subluxation, and muscle activity, no significant changes in AROM were observed after tapping. It is out of our expectation that, along with the significant reduction of pain, the AROM shoulder increased correspondingly. It is known that pain is an inhibiting factor to the neuromuscular activity [7] and the effort of a patient to move. The results might indicate that muscle weakness was a dominant reason for the limited AROM. After 4 weeks of treatment, the taping group showed much greater improvement in AROM than the control group, indicating that kinesiology taping can enhance the recovery of motor function when accompanied with conventional treatment. The effectiveness might be contributed to the reduction of pain which can enhance the initiative of patient to conduct exercise. Furthermore, kinesiology taping seems to be able to activate the neuromuscular function, which is very crucial for the recovery of the motor function in patients after stroke.

4.1. Study Limitations. There were some limitations in the present study that should be noted. First, the characteristics of the patients in this study met specific criteria. For example, only patients whose pain can be reduced by joint reposition were recruited. Second, there was no special examination, such as X-ray or ultrasound, adopted in the study to document the shoulder injury and healing. Third, the sample size was small, and all participants belonged to the same hospital. Finally, only 4 weeks of treatment was conducted, without long-term follow-up data. Hence, the ability to generalize the findings in the present study to the poststroke population is limited.

\section{Conclusion}

The present study revealed the effectiveness of kinesiology taping for HSP. Kinesiology taping might be a good alternative for relieving shoulder pain, improving the AROM, subluxation, and muscle activity of the shoulder in patients with HSP after stroke.

\section{Abbreviations}

HSP: Hemiplegic shoulder pain

AROM: Active range of movement

sEMG: Surface electromyography

NPRS: Numerical pain rating scale.

\section{Conflicts of Interest}

The authors declare that they have no conflicts of interest.

\section{References}

[1] I. Lindgren, A. C. Jönsson, B. Norrving, and A. Lindgren, "Shoulder pain after stroke: a prospective population-based study," Stroke, vol. 38, no. 2, pp. 343-348, 2007.

[2] N. Sayiner Caglar, T. Akin, E. Aytekin et al., "Pain syndromes in hemiplegic patients and their effects on rehabilitation results," Journal of Physical Therapy Science, vol. 28, no. 3, pp. 731-737, 2016.

[3] S. Suethanapornkul, P. S. Kuptniratsaikul, V. Kuptniratsaikul, P. Uthensut, P. Dajpratha, and J. Wongwisethkarn, "Post stroke shoulder subluxation and shoulder pain: a cohort multicenter study," Journal of the Medical Association of Thailand, vol. 91, no. 12, pp. 1885-1892, 2008.

[4] V. M. Parker, D. T. Wade, and R. Langton Hewer, "Loss of arm function after stroke: measurement, frequency, and recovery," International Rehabilitation Medicine, vol. 8, no. 2, pp. 69-73, 1986.

[5] C. Van Ouwenaller, P. M. Laplace, and A. Chantraine, "Painful shoulder in hemiplegia," Archives of Physical Medicine and Rehabilitation, vol. 67, no. 1, pp. 23-26, 1986.

[6] I. Coskun Benlidayi and S. Basaran, "Hemiplegic shoulder pain: a common clinical consequence of stroke," Practical Neurology, vol. 14, no. 2, pp. 88-91, 2014. 
[7] P. W. Hodges, U. F. Ervilha, and T. Graven-Nielsen, "Changes in motor unit firing rate in synergist muscles cannot explain the maintenance of force during constant force painful contractions," Journal of Pain, vol. 9, no. 12, pp. 1169-1174, 2008.

[8] A. Yetisgin, "Clinical characteristics affecting motor recovery and ambulation in stroke patients," Journal of Physical Therapy Science, vol. 29, no. 2, pp. 216-220, 2017.

[9] M. Paci, L. Nannetti, P. Taiti, M. Baccini, J. Pasquini, and L. Rinaldi, "Shoulder subluxation after stroke: relationships with pain and motor recovery," Physiotherapy Research International, vol. 12, no. 2, pp. 95-104, 2007.

[10] C. W. Roy, M. R Sands, L. D. Hill, A. Harrison, and S. Marshall, "The effect of shoulder pain on outcome of acute hemiplegia," Clinical Rehabilitation, vol. 9, no. 1, pp. 21-27, 1995.

[11] Y. Yi, J. S. Shim, K. Kim et al., "Han TR, Prevalence of the rotator cuff tear increases with weakness in hemiplegic shoulder," Annals of Rehabilitation Medicine, vol. 37, no. 4, pp. 471-478, 2013.

[12] S. F. Lo, S. Y. Chen, H. C. Lin, Y. F. Jim, N. H. Meng, and M. J. Kao, "Arthrographic and clinical findings in patients with hemiplegic shoulder pain," Archives of Physical Medicine and Rehabilitation, vol. 84, no. 12, pp. 1786-1791, 2003.

[13] D. G. F. Tavora, R. L. Gama, R. C. Bomfim, M. Nakayama, and C. E. P. Silva, "MRI findings in the painful poststroke shoulder," Clinical Radiology, vol. 65, no. 10, pp. 789-794, 2010.

[14] I. Lindgren and C. Brogardh, "Poststroke shoulder pain and its association with upper extremity sensorimotor function, daily hand activities, perceived participation, and life satisfaction," Physical Medicine and Rehabilitation, vol. 6, no. 9, pp. 781-789, 2014.

[15] A. Barlak, S. Unsal, and K. Kaya, "Poststroke shoulder pain in Turkish stroke patients: relationship with clinical factors and functional outcomes," International Journal of Rehabilitation Research, vol. 32, no. 4, pp. 309-315, 2009.

[16] J. Soo Hoo, T. Paul, J. Chae, and R. D. Wilson, "Central hypersensitivity in chronic hemiplegic shoulder pain," American Journal of Physical Medicine and Rehabilitation, vol. 92, no. 1, pp. 1-13, 2013.

[17] A. Van Bladel, G. Lambrecht, K. M. Oostra, G. Vanderstraeten, and D. Cambier, "A randomized controlled trial on the immediate and long-term effects of arm slings on shoulder subluxation in stroke patient," European Journal of Physical and Rehabilitation Medicine, vol. 53, no. 3, pp. 400-409, 2017.

[18] C. Griffin, "Management of the hemiplegic shoulder complex," Topics in Stroke Rehabilitation, vol. 21, no. 4, pp. 316-318, 2014.

[19] D. Suriya-amarit, C. Gaogasigam, A. Siriphorn, and S. Boonyong, "Effect of interferential current stimulation in management of hemiplegic shoulder pain," Archives of Physical Medicine and Rehabilitation, vol. 95, no. 8, pp. 1441-1446, 2014.

[20] B. Gialanella, P. Benvenuti, and R. Santoro, "The painful hemiplegic shoulder: effects of exercises program according to Bobath," Clinica Terapeutica, vol. 155, no. 11-12, pp. 491-497, 2004.

[21] D. Stolzenberg, G. Siu, and E. Cruz, "Current and future interventions for glenohumeral subluxation in hemiplegia secondary to stroke," Topics in Rehabilitation Stroke, vol. 19, no. 5, pp. 444-456, 2012.
[22] M. Murie-Fernandez, M. Carmona Iragui, V. Gnanakumar, M. Meyer, N. Foley, and R. Teasell, "Painful hemiplegic shoulder in stroke patients: causes and management," Neurologia, vol. 27, no. 4, pp. 234-244, 2012.

[23] Y. C. Huang, K. H. Chang, T. H. Liou, C. W. Cheng, L. F. Lin, and S. W. Huang, "Effects of Kinesio taping for stroke patients with hemiplegic shoulder pain: a double-blind, randomized, placebo-controlled study," Journal of Rehabilitation of Medcine, vol. 49, no. 3, pp. 208-215, 2015.

[24] C. Y. Huang, T. H. Hsieh, S. C. Lu, and F. C. Su, "Effect of the Kinesio tape to muscle activity and vertical jump performance in healthy inactive people," BioMedical Enginering Online, vol. 10, no. 1, p. 70, 2011.

[25] E. Kaya, M. Zinnuroglu, and I. Tugcu, "Kinesio taping compared to physical therapy modalities for the treatment of shoulder impingement syndrome," Clinical Rheumatology, vol. 30, no. 2, pp. 201-207, 2011.

[26] M. A. Ferreira-Valente, J. L. Pais-Ribeiro, and M. P. Jensen, "Validity of four pain intensity rating scales," Pain, vol. 152, no. 10, pp. 2399-2404, 2011.

[27] J. D. Pandian, P. Kaur, R. Arora et al., "Shoulder taping reduces injury and pain in stroke patients: randomized controlled trial," Neurology, vol. 80, no. 6, pp. 528-532, 2013.

[28] A. Griffin and J. Bernhardt, "Strapping the hemiplegic shoulder prevents development of pain during rehabilitation: a randomized controlled trial," Clinical Rehabilitation, vol. 20, no. 4, pp. 287-295, 2006.

[29] J. Gonzalez-Iglesias, C. Fernandez-de-las-Penas, J. Cleland, P. Huijbregts, and M. R. Gutierrez0vega, "Short-term effects of cervical kinesio taping on pain and cervical range of motion in patients with acute whiplash injury: a randomized clinical trial," Journal of Orthopaedic and Sports Physical Therapy, vol. 39, no. 7, pp. 515-521, 2009.

[30] M. Paoloni, A. Bernetti, G. Fratocchi et al., "Kinesio Taping applied to lumbar muscles influences clinical and electromyographic characteristics in chronic low back pain patients," European Journal of Physical and Rehabilitation Medicine, vol. 47, no. 2, pp. 237-244, 2011.

[31] M. D. Thelen, J. A. Dauber, and P. D. Stoneman, "The clinical efficacy of Kinesio Tape for shoulder pain: a randomized, double-blinded, clinical trial," Journal of Orthopaedic and Sports Physical Therapy, vol. 38, no. 7, pp. 389-395, 2008.

[32] E. Jaraczewska and C. Long, "Kinesio ${ }^{\circledR}$ taping in stroke: improving functional use of the upper extremity in hemiplegia," Topics in Stroke Rehabilitation, vol. 13, no. 3, pp. 31-42, 2006.

[33] J. U. McCarthy Persson, A. C. B. Hooper, and H. E. Fleming, "Repeatability of skin displacement and pressure during inhibitory vastus lateralis muscle taping," Manual Therapy, vol. 12, no. 1, pp. 17-21, 2007.

[34] J. T. Han, J. H. Lee, and C. H. Yoon, "The mechanical effect of kinesiology tape on rounded shoulder posture in seated male workers: a single-blinded randomized controlled pilot study," Physiotherapy Theory and Practice, vol. 31, no. 2, pp. 120-125, 2015.

[35] J. H. Lee, W. G. Yoo, and G. Hwang-Bo, "The immediate effect of anterior pelvic tilt taping on pelvic inclination," Journal of Physical Therapy Science, vol. 23, no. 2, pp. 201-203, 2011.

[36] K. Dieruf, J. L. Poole, C. Gregory, E. J. Rodriguez, and C. Spizman, "Comparative effectiveness of the GivMohr sling in subjects with flaccid upper limbs on subluxation through radiologic analysis," Archives of Physical Medicine and Rehabilitation, vol. 86, no. 12, pp. 2324-2329, 2005.

[37] S. H. Han, T. Kim, S. H. Jang et al., "The effect of an arm sling on energy consumption while walking in hemiplegic patients: 
a randomized comparison," Clinical Rehabilitation, vol. 25, no. 1, pp. 36-42, 2011.

[38] M. Niessen, T. Janssen, C. Meskers, P. Koppe, M. Konijnenbelt, and D. Veeger, "Kinematics of the contralateral and ipsilateral shoulder: a possible relationship with post-stroke shoulder pain," Journal of Rehabilitation Medicine, vol. 40, no. 6, pp. 482-486, 2008.

[39] R. W. Bragg, J. M. Macmahon, E. K. Overom et al., "Failure and fatigue characteristics of adhesive athletic tape," Medicine and Science in Sports and Exercise, vol. 34, no. 3, pp. 403-410, 2002.

[40] K. Macgregor, S. Gerlach, R. Mellor, and P. W. Hodges, "Cutaneous stimulation from patella tape causes a differential increase in vasti muscle activity in people with patellofemoral pain," Journal of Orthopaedic Research, vol. 23, no. 2, pp. 351-358, 2005.

[41] M. H. Kang, S. H. Choi, and J. S. Oh, "Postural taping applied to the low back influences kinematics and EMG activity during patient transfer in physical therapists with chronic low back pain," Journal of Electromyography and Kinesiology, vol. 23, no. 4, pp. 787-793, 2013.

[42] J. J. Lin, C. J. Hung, and P. L. Yang, "The effects of scapular taping on electromyographic muscle activity and proprioception feedback in healthy shoulders," Journal of Orthopaedic Research, vol. 29, no. 1, pp. 53-57, 2011.

[43] C. G. Ryan and P. J. Rowe, "An electromyographical study to investigate the effects of patellar taping on the vastus medialis/ vastus lateralis ratio in asymptomatic participants," Physiotherapy Theory and Practice, vol. 22, no. 6, pp. 309-315, 2006. 


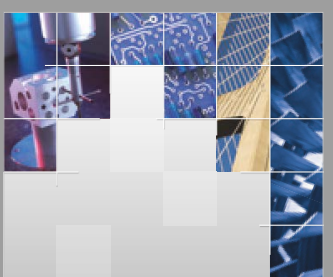

\section{Enfincering}
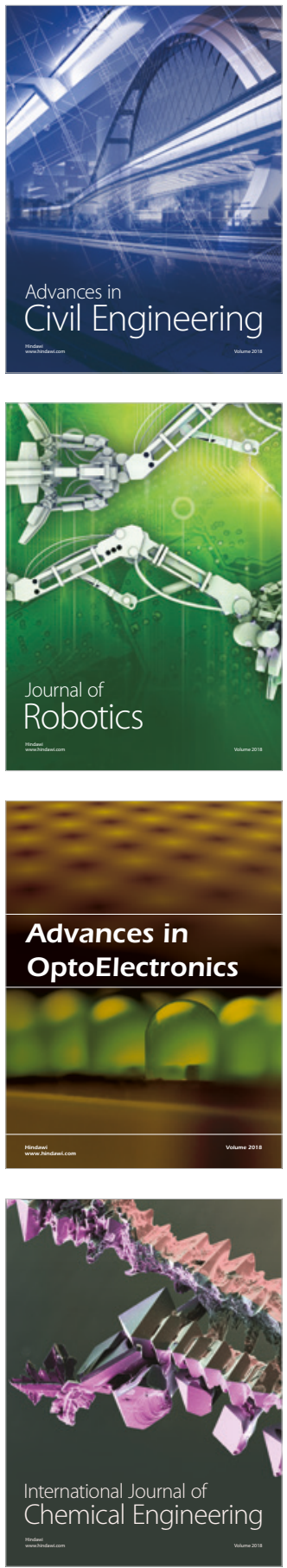

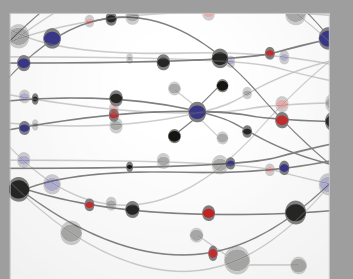

\section{Rotating \\ Machinery}

The Scientific World Journal

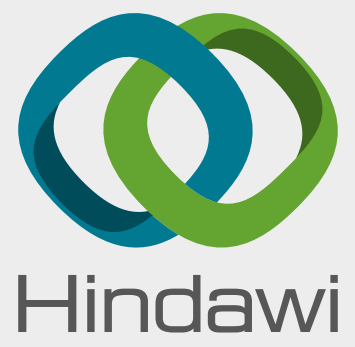

Submit your manuscripts at

www.hindawi.com
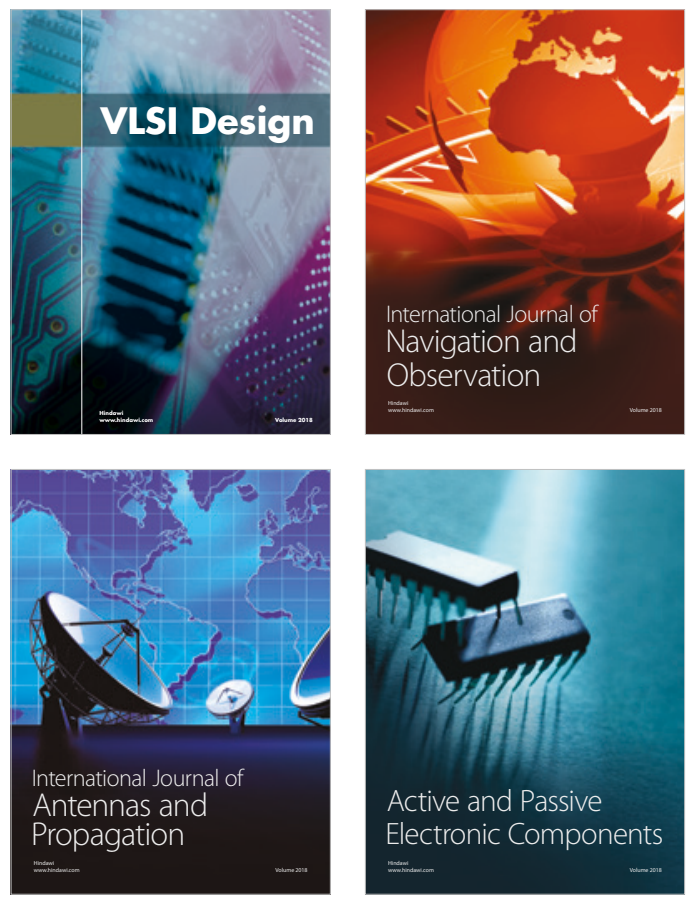
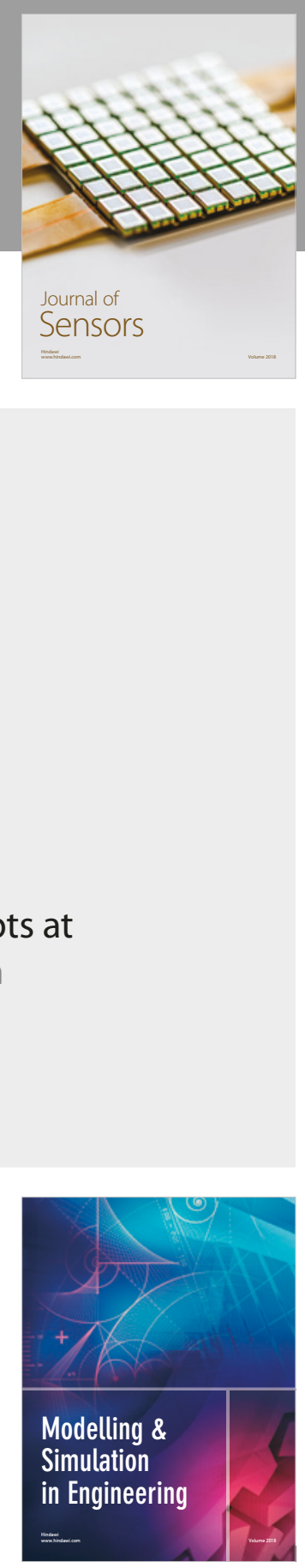

\section{Advances \\ Multimedia}
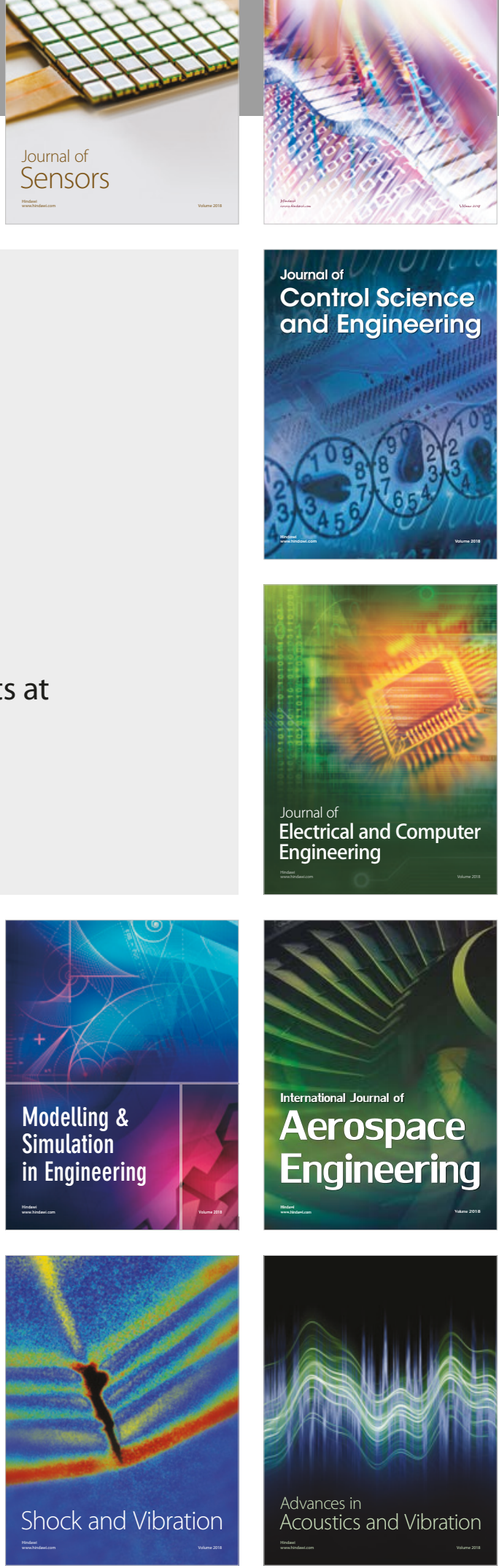